\title{
Genetic variability of quantitative traits in Drosophila melanogaster (fruit fly) natural populations: analysis of wild-living flies and of several laboratory generations
}

\author{
PATRICIA GIBERT*, BRIGITTE MORETEAU, J EAN-CLAUDE MORETEAU† \& J EAN R. \\ DAVID \\ CNRS, Laboratoire Populations, Génétique et Evolution, 91198 Gif sur Yvette Cedex, France and †Université de \\ Metz, Laboratoire de Démoécologie, 1, rue des Récollets, 57000 Metz Cedex, France
}

\begin{abstract}
Wild-collected Drosophila melanogaster females were isolated in culture vials to initiate isofemale lines in the laboratory. Wing length and body pigmentation (thorax, abdominal segments 5, 6 and 7 and their sum) were measured in wild flies and in successive laboratory generations. Heritability was estimated by parent-offspring regression between wild-living flies and laboratory progeny or by calculating intraclass correlation between lines in each laboratory generation. For wing length, phenotypic variability was much higher in nature than in the laboratory, and the estimated heritability with parent-offspring regression (0.14) was significant but quite low. Within laboratory generations, intraclass correlation was much higher, on average 0.34. For pigmentation characteristics, variability in nature was similar to that measured in the laboratory. Parent-offspring regressions produced significant and high heritability values (range 0.40-0.62), except for abdomen segment 5. Intraclass correlations were also significantly greater than zero for all traits (range $0.22-0.47$ ), including segment 5 . The stability of the lines over successive laboratory generations was shown by the stability of the overall mean and by a strong positive correlation between family means of successive generations. The correlation across generations demonstrates a genetic repeatability of the trait and should be useful in experiments using isofemale lines.
\end{abstract}

Keywords: body pigmentation, genetic repeatability, heritability, intraclass correlation, isofemale lines, wing length.

\section{Introduction}

A condition for adaptive evolution in a natural population is the presence of additive genetic variation for the trait under consideration. In the case of Drosophila, regular latitudinal clines have been described for various traits, and they are generally assumed to be a consequence of climatic selection and adaptation (David et al., 1983; Parsons, 1983; David \& Capy, 1988; Hoffman \& Parsons, 1991; Capy et al., 1993; Partridge et al., 1994). A recurring observation in several species is a genetic increase in body size with latitude. Wild-living flies, however, exhibit a huge phenotypic variance in body size (David et al., 1980; Coyne \& Beecham, 1987; Imasheva et al., 1994; Moreteau et al., 1995), which

*Correspondence. is caused by environmental heterogeneity during development. For that reason, the genetic characteristics of natural populations, including geographical clines and heritability, are generally investigated in the laboratory under controlled conditions, in order to minimize the environmental variance and provide better genetic information. This procedure raises several problems that are generally not addressed, such as the choice of laboratory conditions and the generation to be investigated. For keeping a natural population in the laboratory, two procedures can be used: either a large population is maintained in a single cage (e.g. Partridge et al., 1994) or isofemale lines are established and kept separately (Parsons, 1983; Capy et al., 1993).

Two opposite processes are, however, likely to occur in successive laboratory generations. Genetic drift in a neutral environment should progressively 
increase the divergence between lines and thus the genetic heterogeneity. If a mass population is kept, drift will be slower but will occur anyway after a few years. In contrast, if the laboratory conditions are not neutral for the trait investigated, some kind of laboratory adaptation will be observed. This possible adaptation will shift the overall mean from the initial value to some new equilibrium. In the case of parallel lines, adaptation should induce a convergence towards the selected optimum and thus reduce their genetic heterogeneity.

Numerous examples of both drift and adaptation have been described for discrete genetic markers, including phenotypic mutants, chromosome rearrangements or allozymes (Dobzhansky, 1970; Lewontin, 1974; Merrel, 1981; Van Delden, 1982; Delpuech et al., 1993). Data on quantitative traits are, on the other hand, less well documented. An increased divergence of ovariole number among laboratory strains was interpreted as a consequence of genetic drift (Bocquet et al., 1973). A classical example of laboratory temperature adaptation refers to strains kept at various temperatures, which, after a few years, exhibited a systematic divergence, i.e. a smaller size at higher temperature and a larger size at lower temperature (David et al., 1983; Cavicchi et al., 1985; Partridge et al., 1994).

In the present study, the genetic variability of wild-living flies and their laboratory progeny was investigated over several generations. Two kinds of quantitative traits were measured: wing length (related to body size) and body pigmentation (thorax and abdomen) in females. In nature, wing length exhibited a huge phenotypic variance, as expected from previous results. Pigmentation traits were less variable and highly heritable. Genetic variability among isofemale lines in the laboratory was high for all traits and stable over generations. The genetic stability of the lines was also demonstrated by a high positive correlation (genetic repeatability) between family means over successive generations. The techniques implemented here should be a model for analysing experimental adaptations to different environments.

\section{Materials and methods}

A wild-living D. melanogaster population was collected with banana traps in Cotonou (Republic of Benin, Africa). Adults were put in sucrose-agar vials and sent by air mail to France. On arrival, females were analysed for morphometrical traits and then isolated at $25^{\circ} \mathrm{C}$ in culture vials containing a killed yeast medium to establish isofemale lines.
Among 60 females that were isolated, only 37 produced sufficient progeny (at least 10 females) to be analysed further.

From each isofemale line, 10 laboratory-grown females were taken randomly and measured. Total wing length from thorax articulation to the tip of the third longitudinal vein was measured with a micrometer under a binocular microscope. The dark pigmentation on the last three abdomen segments (5, 6 and 7) was estimated visually, and their sum was also calculated (see David et al., 1990). For each segment, 11 classes ranging from 0 (no dark pigment) to 10 (segment completely dark) were used. The intensity of pigmentation of a trident pattern on the mesonotum was also estimated. In that case, four phenotypic classes from 0 (no visible trident) to 3 (dark trident) were used (see David et al., 1985). The 10 measured females were then associated with 10 randomly taken males and used for producing the next generation. Egg laying of the 10 pairs was limited to a few hours, and crowding effects were avoided by using a killed yeast highnutrient medium.

Ten females of the next generation were again taken at random, measured and used as parents of the following generation. The first three laboratory generations (G1, G2 and G3) were investigated in this way. Females of the fourth generation were not investigated but the method used for propagating each line (i.e. 10 randomly chosen parental pairs) was kept the same. The G5 generation was again investigated and, finally, the G9 generation.

\section{Results}

Mean values of parental flies and laboratory progeny

Basic data concerning the six traits investigated are given in Table 1, and the variability among lines is illustrated (Fig. 1) for two traits, the abdomen pigmentation (sum) and wing length.

The two parental samples ( $\mathrm{P} 1$, the whole sample, and $\mathrm{P} 2$, the mothers of isofemale lines) were similar for all traits and not statistically different. The P2 females, which were chosen because of their higher progeny number, did not differ from the whole natural sample. Compared with laboratory-grown flies, these wild-living females were different for all traits. The major difference concerned size, i.e. wing length. Wild-living females were on average smaller and also much more variable (see Fig. 1). The phenotypic variance in wild-living females was 273.8 against 31.3 in laboratory-grown flies, i.e. 
Table 1 Mean values ( \pm standard error) of morphometrical traits in wild-collected female Drosophila melanogaster (P1 and P2) and laboratory generations of 37 isofemale lines

\begin{tabular}{lrrrrrrr}
\hline & \multicolumn{1}{c}{ P1 } & \multicolumn{1}{c}{ P2 } & G1 & \multicolumn{1}{c}{ G2 } & G3 & \multicolumn{1}{c}{ G5 } & G9 \\
& Total (70) & \multicolumn{1}{c}{ (37) } & & & & & \\
\hline Trident & $0.19 \pm 0.05$ & $0.16 \pm 0.06$ & $0.05 \pm 0.01$ & $0.08 \pm 0.01$ & $0.05 \pm 0.01$ & $0.04 \pm 0.01$ & $0.04 \pm 0.01$ \\
Segment 5 & $3.11 \pm 0.08$ & $2.92 \pm 0.09$ & $2.95 \pm 0.04$ & $3.06 \pm 0.04$ & $2.84 \pm 0.04$ & $3.17 \pm 0.03$ & $2.96 \pm 0.04$ \\
Segment 6 & $5.71 \pm 0.18$ & $5.54 \pm 0.25$ & $6.67 \pm 0.07$ & $6.65 \pm 0.08$ & $6.46 \pm 0.08$ & $7.07 \pm 0.08$ & $6.74 \pm 0.08$ \\
Segment 7 & $3.37 \pm 0.25$ & $3.22 \pm 0.34$ & $4.94 \pm 0.11$ & $4.97 \pm 0.12$ & $4.66 \pm 0.12$ & $5.35 \pm 0.12$ & $4.78 \pm 0.12$ \\
Sum & $12.20 \pm 0.43$ & $11.68 \pm 0.56$ & $14.56 \pm 0.19$ & $14.68 \pm 0.19$ & $13.97 \pm 0.20$ & $15.58 \pm 0.19$ & $14.49 \pm 0.21$ \\
Wing & $224.97 \pm 1.98$ & $225.84 \pm 2.88$ & $253.22 \pm 0.26$ & $244.94 \pm 0.31$ & $252.08 \pm 0.32$ & $254.35 \pm 0.29$ & $251.10 \pm 0.26$ \\
\hline
\end{tabular}

For laboratory generations, standard errors were calculated from the mean values of the 37 lines. P1, whole sample of 70 wild-living females. P2, subsample of 37 females whose progeny was investigated. G1, first laboratory generation; G2-G9, successive laboratory generations of the same lines.

Trident, pigmentation score of the thoracic trident (possible range 0-3).

Segments 5, 6 and 7, pigmentation score of abdomen segments 5, 6 and 7 (possible range 0-10).

Sum, sum for each fly of the three abdomen segments (range 0-30).

Wing, wing length in $\mathrm{mm} \times 100$.

8.8 times higher. The thoracic trident, poorly marked in this tropical population, was darker in wild-living flies than in the laboratory progeny anyway. The reverse was true for abdomen pigmentation, which was darker in laboratory flies than in wild-living ones, especially for segments 6 and 7 . Interestingly, the phenotypic variability of females in nature was close to the variability observed in the laboratory (see Fig. 1). For the sum of the three segments, the phenotypic variance in nature was 12.8 against 14.2 in the laboratory.

The wild population of Cotonou investigated here was living at a temperature of more than $25^{\circ} \mathrm{C}$ (range $27-29^{\circ} \mathrm{C}$ ). The average wing length, which is shorter than in laboratory generations, may be explained by both poor feeding conditions and higher developmental temperature. This higher temperature also explains the overall lighter pigmentation of the abdomen segments (David et al., 1990).

\section{Variability among lines and generations in the laboratory}

Morphometrical data of generations G1-G9 were submitted to a nested ANOVA (not shown). A highly significant line effect was observed for all traits, suggesting their genetic heterogeneity. For pigmentation characters, no significant variation was found between generations except a slight effect for segment 5. A significant heterogeneity between generations was, however, shown for wing length. As visualized in Fig. 1, this effect is accounted for by a general decrease in wing length of all lines in G2.
Excluding the $\mathrm{G} 2$ data led to homogeneity among the four other generations (G1, G3, G5 and G9). The reasons why $\mathrm{G} 2$ values were consistently smaller probably correspond to some uncontrolled rearing accident.

Genetic variation between lines was investigated further by calculating, for each generation and each trait, the coefficient of intraclass correlation, $t$ (Table 2). These values were then submitted to ANOvA (not shown), which showed a highly significant trait effect $\left(F_{5,20}=12.09 ; P<0.001\right)$ and a slight generation effect $\left(F_{4,20}=3.02 ; P<0.05\right)$. The trait effect is obvious from Table 2: the coefficient of intraclass correlation is minimum for the trident $(0.22 \pm 0.02)$, intermediate for wing length $(0.34 \pm 0.03)$ and maximum for abdomen segments 6 and 7 and for the sum (range 0.41-0.48).

The significant generation effect suggests a slight increase of $t$ with time: the mean value in G9 is 0.44 , whereas it is 0.34 for the previous generations. Such a phenomenon might result from genetic drift occurring among lines in the laboratory. The problem was further investigated by calculating the between-line variance, which estimates the genetic variance, and the within-line variance, which is mainly attributable to environmental effects (Table 3). For each trait, variances were submitted to a Bartlett's homogeneity test, and no significant differences among generations were observed. The slight increase in genetic variability over time, which is evident in the whole set of intraclass correlations, is too small to be demonstrated when considering the variances of each trait separately.

(C) The Genetical Society of Great Britain, Heredity, 80, 326-335. 

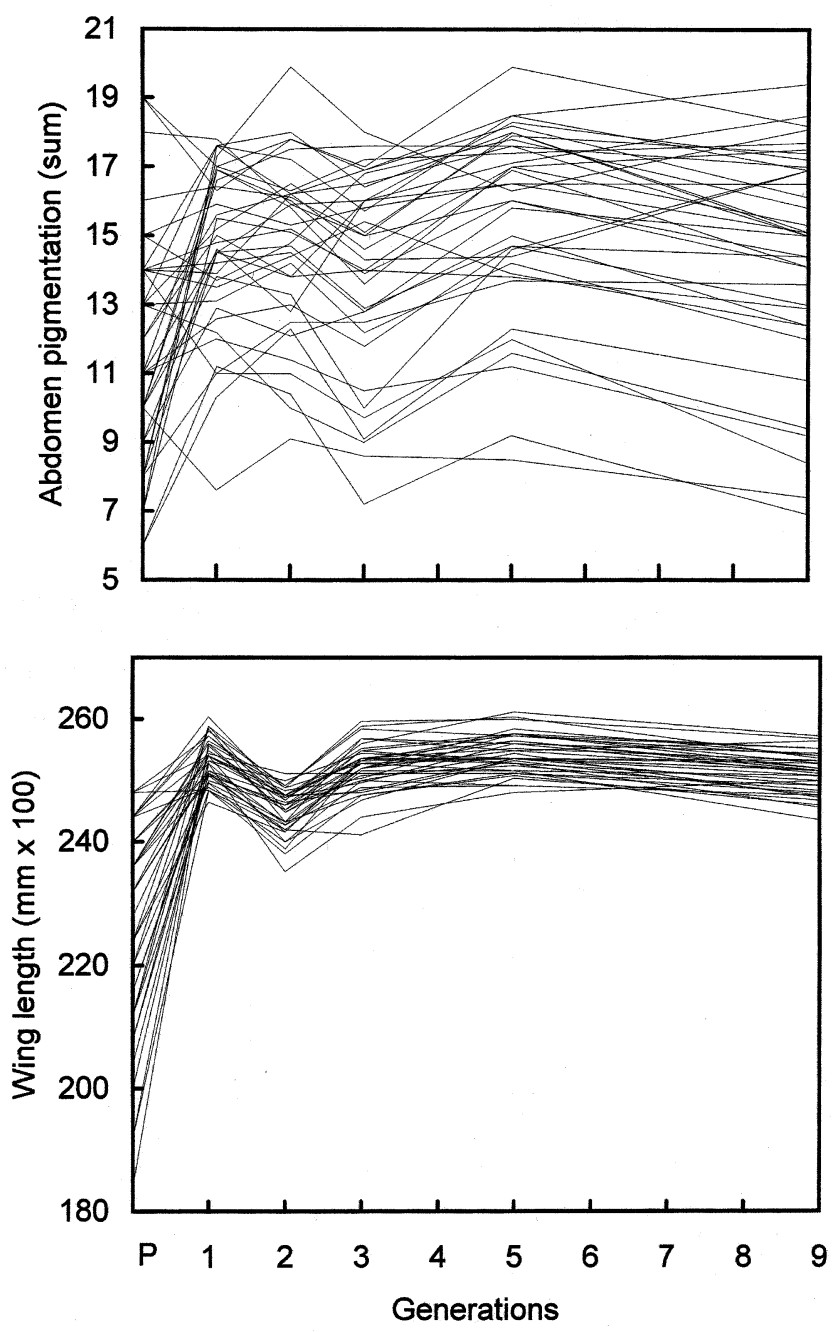

Fig. 1 Mean values of parental Drosophila melanogaster (P) and laboratory generations (G1-G9) for the sum of abdomen pigmentation and wing length (37 lines).
Correlation and regression between wild-living females and laboratory progeny

Regression coefficients between wild parents and laboratory offspring are a classical means of estimating heritability (Falconer, 1989). In the present case, because one sex only (mother-daughters regression) was considered, heritability should be twice the regression coefficient. To be applied correctly, this formula implies that parents and offspring developed in the same environment and exhibit similar phenotypic variances, a condition which obviously does not apply for wing length. In this case, it may be suspected that additive genetic variance in nature is different from the same parameter in the laboratory, and various techniques can be used to approach this problem (Riska et al., 1989). The classical mother-daughter regression, $b$ (Table 4), was calculated, as well as two other quantities suggested by Riska et al. (1989) $-\gamma^{2} h^{2}$ and $h^{2} v-$ both of which could provide estimates of heritability in nature. As several laboratory generations were available, it was possible to correlate wild-living females with their $F_{1}$ progeny and also with successive generations (Table 4 ). The parent $-\mathrm{F}_{1}$ regression was very low (0.02) and not significantly different from zero. The same coefficient, calculated with more distant generations, tended to increase, becoming significantly greater than zero. Such variations are difficult to explain, except by random fluctuations between samplings. The best estimate of the regression is thus the mean value, $0.07 \pm 0.01$, significantly greater than zero and suggesting a heritability of $0.14 \pm 0.02$.

The quantity $\gamma^{2} h^{2}=4 b^{2}\left(V_{\mathrm{PN}} / V_{\mathrm{AL}}\right)$ (where $V_{\mathrm{PN}}$ is the phenotypic variance in nature and $V_{\mathrm{AL}}$ is the additive genetic variance in the laboratory) proved

Table 2 Values of intraclass correlations calculated for six different morphometrical traits in five laboratory generations (G1-G9) of Drosophila melanogaster

\begin{tabular}{lccccccc}
\hline Trait & G1 & G2 & G3 & G5 & G9 & Mean & SE \\
\hline Trid. & 0.24 & 0.20 & 0.14 & 0.25 & 0.29 & 0.22 & 0.02 \\
S5 & 0.27 & 0.28 & 0.22 & 0.22 & 0.34 & 0.27 & 0.02 \\
S6 & 0.38 & 0.35 & 0.29 & 0.49 & 0.54 & 0.41 & 0.04 \\
S7 & 0.39 & 0.38 & 0.56 & 0.48 & 0.57 & 0.48 & 0.04 \\
Sum & 0.39 & 0.38 & 0.52 & 0.49 & 0.56 & 0.47 & 0.03 \\
Wing & 0.45 & 0.30 & 0.33 & 0.24 & 0.36 & 0.34 & 0.03 \\
Mean & 0.35 & 0.32 & 0.34 & 0.36 & 0.44 & 0.36 & \\
SE & 0.03 & 0.03 & 0.07 & 0.06 & 0.05 & 0.04 & \\
\hline
\end{tabular}

Trid., trident; S5, S6, S7, abdominal segments 5, 6 and 7; sum, sum of segments 5,6 and 7. 
Table 3 Variances among lines and within lines (laboratory generations) of Drosophila melanogaster

\begin{tabular}{lccccc}
\hline Trait & G1 & G2 & G3 & G5 & G9 \\
\hline Variance among lines & & & & & \\
$\quad$ Trid. & 0.011 & 0.015 & 0.007 & 0.010 & 0.011 \\
S5 & 0.126 & 0.140 & 0.126 & 0.097 & 0.199 \\
S6 & 0.797 & 0.739 & 0.964 & 1.061 & 1.441 \\
S7 & 1.889 & 1.951 & 2.798 & 2.433 & 3.200 \\
Sum & 5.139 & 5.164 & 7.492 & 6.513 & 9.531 \\
Wing & 10.87 & 10.86 & 12.86 & 7.72 & 9.29 \\
Variance within lines & & & & & \\
Trid. & 0.036 & 0.059 & 0.047 & 0.031 & 0.026 \\
S5 & 0.342 & 0.362 & 0.437 & 0.350 & 0.387 \\
S6 & 1.292 & 1.377 & 2.352 & 1.087 & 1.220 \\
S7 & 2.911 & 3.259 & 2.240 & 2.594 & 2.441 \\
Sum & 8.150 & 8.539 & 6.837 & 6.892 & 7.383 \\
Wing & 13.14 & 25.07 & 26.34 & 24.19 & 16.79 \\
\hline
\end{tabular}

Trid., trident; S5, S6, S7, abdominal segments 5, 6 and 7; sum, sum of segments 5,6 and 7.

to be useless, with very high values, one of them greater to 1 (Table 4 ). The quantity $h^{2} v=V_{\mathrm{AL}} / V_{\mathrm{PN}}$ was always very low, ranging between 0.025 and 0.042. As indicated by Riska et al. (1989), it is a lower bound of heritability in nature.

The difficulty of heteroscedasticity did not exist for pigmentation traits, and the mother-daughter regression appears as a convenient estimate of heritability. In several cases (segments 6, 7 and the sum), a tendency existed for an increased regression coefficient when more distant progeny generations were considered. We suggest again that the average value should be considered as the best estimate of heritability. Surprisingly, heritability for segment 5 seems to be very low and not different from zero. For other traits, values range between 0.20 and 0.31 , corresponding to heritabilities from 0.40 to 0.62 .

\section{Correlation between laboratory generations: genetic repeatability}

A highly significant line effect in ANOVA (not shown) as well as the graphs in Fig. 1 show that the mean value of each line remained quite stable, or repeatable, over successive generations. A way to estimate this genetic repeatability is to calculate the correlation between the mean values of the lines in different generations (Table 5). All coefficients were

Table 4 Relationship between wild-living parental female Drosophila melanogaster and laboratory progeny in successive generations

\begin{tabular}{|c|c|c|c|c|c|c|c|}
\hline & & G1 & G2 & G3 & G5 & G9 & Mean \pm SE \\
\hline Trident & $b$ & $0.22 \pm 0.04$ & $0.26 \pm 0.05$ & $0.18 \pm 0.04$ & $0.19 \pm 0.04$ & $0.15 \pm 0.05$ & $0.20 \pm 0.02$ \\
\hline Segt 5 & $b$ & $0.08 \pm 0.12$ & $0.12 \pm 0.13$ & $0.18 \pm 0.12$ & $-0.07 \pm 0.11$ & $-0.10 \pm 0.15$ & $0.04 \pm 0.05$ \\
\hline Segt 6 & $b$ & $0.23 \pm 0.10$ & $0.19 \pm 0.10$ & $0.27 \pm 0.11$ & $0.30 \pm 0.11$ & $0.30 \pm 0.13$ & $0.26 \pm 0.02$ \\
\hline Segt 7 & $b$ & $0.18 \pm 0.11$ & $0.26 \pm 0.11$ & $0.28 \pm 0.13$ & $0.28 \pm 0.12$ & $0.29 \pm 0.14$ & $0.23 \pm 0.05$ \\
\hline Sum & $b$ & $0.20 \pm 0.11$ & $0.24 \pm 0.11$ & $0.35 \pm 0.13$ & $0.36 \pm 0.12$ & $0.37 \pm 0.14$ & $0.31 \pm 0.03$ \\
\hline Wing & $b$ & $0.02 \pm 0.03$ & $0.06 \pm 0.03$ & $0.08 \pm 0.03$ & $0.09 \pm 0.03$ & $0.07 \pm 0.03$ & $0.07 \pm 0.01$ \\
\hline Mean \pm SE & & $0.14 \pm 0.04$ & $0.20 \pm 0.03$ & $0.23 \pm 0.04$ & $0.25 \pm 0.04$ & $0.24 \pm 0.05$ & \\
\hline Wing & $h^{2} \gamma^{2}$ & 0.045 & 0.406 & 0.610 & 1.285 & 0.646 & \\
\hline Wing & $h^{2} v$ & 0.035 & 0.035 & 0.042 & 0.025 & 0.030 & \\
\hline
\end{tabular}

For calculating the mean value within generations, data of segment five were excluded. $b$, parent-offspring regression; the quantities $h^{2} \gamma^{2}$ and $h^{2} v$ are defined in the text. 
Table 5 Coefficients of correlation (genetic repeatability) between mean values of the 37 isofemale lines of Drosophila melanogaster in different generations

\begin{tabular}{lcccccc}
\hline Generation interval & S5 & S6 & S7 & Sum & Trident & Wing \\
\hline G1-G2 & 0.77 & 0.86 & 0.84 & 0.88 & 0.82 & 0.53 \\
G1-G3 & 0.84 & 0.74 & 0.81 & 0.80 & 0.76 & 0.56 \\
G1-G5 & 0.67 & 0.75 & 0.80 & 0.81 & 0.61 & 0.39 \\
G1-G9 & 0.65 & 0.80 & 0.74 & 0.80 & 0.77 & 0.54 \\
G2-G3 & 0.71 & 0.81 & 0.87 & 0.86 & 0.63 & 0.52 \\
G2-G5 & 0.54 & 0.80 & 0.79 & 0.81 & 0.44 & 0.48 \\
G2-G9 & 0.51 & 0.87 & 0.74 & 0.82 & 0.53 & 0.42 \\
G3-G5 & 0.69 & 0.87 & 0.86 & 0.88 & 0.66 & 0.69 \\
G3-G9 & 0.63 & 0.85 & 0.77 & 0.83 & 0.73 & 0.62 \\
G5-G9 & 0.64 & 0.91 & 0.85 & 0.88 & 0.73 & 0.76 \\
Mean & 0.67 & 0.83 & 0.81 & 0.84 & 0.67 & 0.55 \\
SE & 0.03 & 0.02 & 0.02 & 0.01 & 0.04 & 0.04 \\
\hline
\end{tabular}

Significance threshold values are $0.325(P=0.05)$ and $0.519(P<0.001)$.

significant, demonstrating a good repeatability of all traits. Significant differences were, however, found between traits (ANOvA, not shown). The least repeatable, or least stable, are wing length $(r=0.55)$, thoracic trident and abdomen segment $5(r=0.67)$, whereas segments 6,7 and the sum exhibit correlations greater than 0.8 (Fig. 2).

It was expected that, because of drift, the repeatability or predictability of the isofemale line characteristics would decrease with the generation interval. A significant but slight effect was detected with ANOVA: the average correlation for all traits was 0.76 when the generation interval was one or two and decreased to 0.69 for longer intervals of seven or eight generations. But the decrease was quite slow: values observed in the various lines at $\mathrm{G} 1$ are still good predictors of values found in G9 $\left(r^{2}=0.48\right)$.
It might be assumed that the parent-offspring regression technique could also be used with isofemale mean values for estimating heritabilities. In fact, this method does not apply, because we are dealing with a group of parents and a group of offspring. If two successive generations are considered, the expectation of the mean in the second generation is identical to that of the first, and variations will be caused by sampling and by uncontrolled environmental effects. This was verified by calculating mean square regressions for the first three generations, i.e. $\mathrm{G} 2=f(\mathrm{G} 1)$ and $\mathrm{G} 3=f(\mathrm{G} 2)$. Regression coefficients for the various traits varied between 0.47 and 1.01, with an average value of $0.80 \pm 0.05(n=12)$. To give equal weight to each generation, independently of their order, the bivariate distributions were characterized by their major
Fig. 2 Relationship between mean values of isofemale lines of Drosophila melanogaster over different laboratory generations. Sum, sum of last abdominal segments (correlation G1-G2). Wing, wing length in $\mathrm{mm} \times 100$ (correlation G1-G3). For each graph, the continuous line is the major axis. $r=$ correlation between generations.
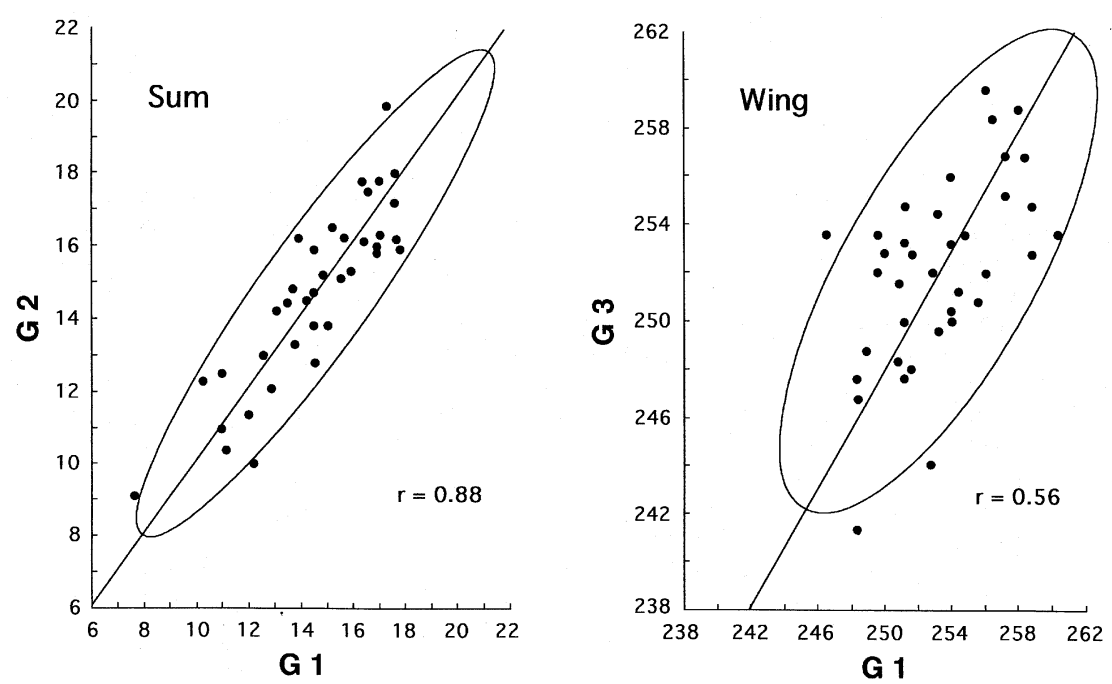
axes. Values were calculated for G1-G2 and G2-G3: they ranged between 0.85 and 1.34 with a mean of $1.027 \pm 0.044(n=12)$. Examples of bivariate distributions are given in Fig. 2.

If the repeatability between generations depends mainly on sampling effects among flies of the same line, it should be influenced by the number of flies measured in each line. In the present case, 10 females were taken for calculating average line values. How the correlation varied according to the number of flies taken from each line by a bootstrap technique was analysed. Results for two characters (sum and wing length) and four pairs of generations are shown in Fig. 3. In all cases, the correlation between family means increased with the sample size. For a highly heritable trait (sum of abdomen pigmentation), a fairly high correlation $(r=0.45 \pm$ 0.03 ) is already observed by taking only one female from each line. Increasing the sample size increases the correlation. With five females per line, the average $r$ becomes $0.79 \pm 0.02$, not much less than the final value obtained with 10 flies $(r=0.88 \pm 0.01)$. The curves in Fig. 3 suggest an asymptotic value of $r$ for bigger sample sizes that would remain lower than 1 . This is because the mean values of successive generations are not expected to be identical, owing to the sampling effect in the choice of parents.

For a less heritable trait (wing length), correlations between family means were much lower: average values with one, five and 10 flies per line were $0.22,0.53$ and 0.63 , respectively.

In practice, two or three flies measured from each line would be sufficient to demonstrate a convenient genetic repeatability for pigmentation. At least five flies would be necessary to achieve the same goal for wing length.

\section{Discussion}

In agreement with all previous investigations (David et al., 1980; Coyne \& Beecham, 1987; Imasheva et al., 1994; Moreteau et al., 1995), our data confirmed that size variations (i.e. wing length) are much greater in nature than in laboratory flies. Because the mean values of the investigated populations are not the same and, more importantly, because the way wing length is measured varies according to investigations, comparisons of variabilities can be made only with a relative estimate, the coefficient of variation $(\mathrm{CV})$. Average values of wing length $\mathrm{CVs}$ from three papers in which several populations of $D$. melanogaster were investigated both in nature and in the laboratory are given in Table 6 . In the present
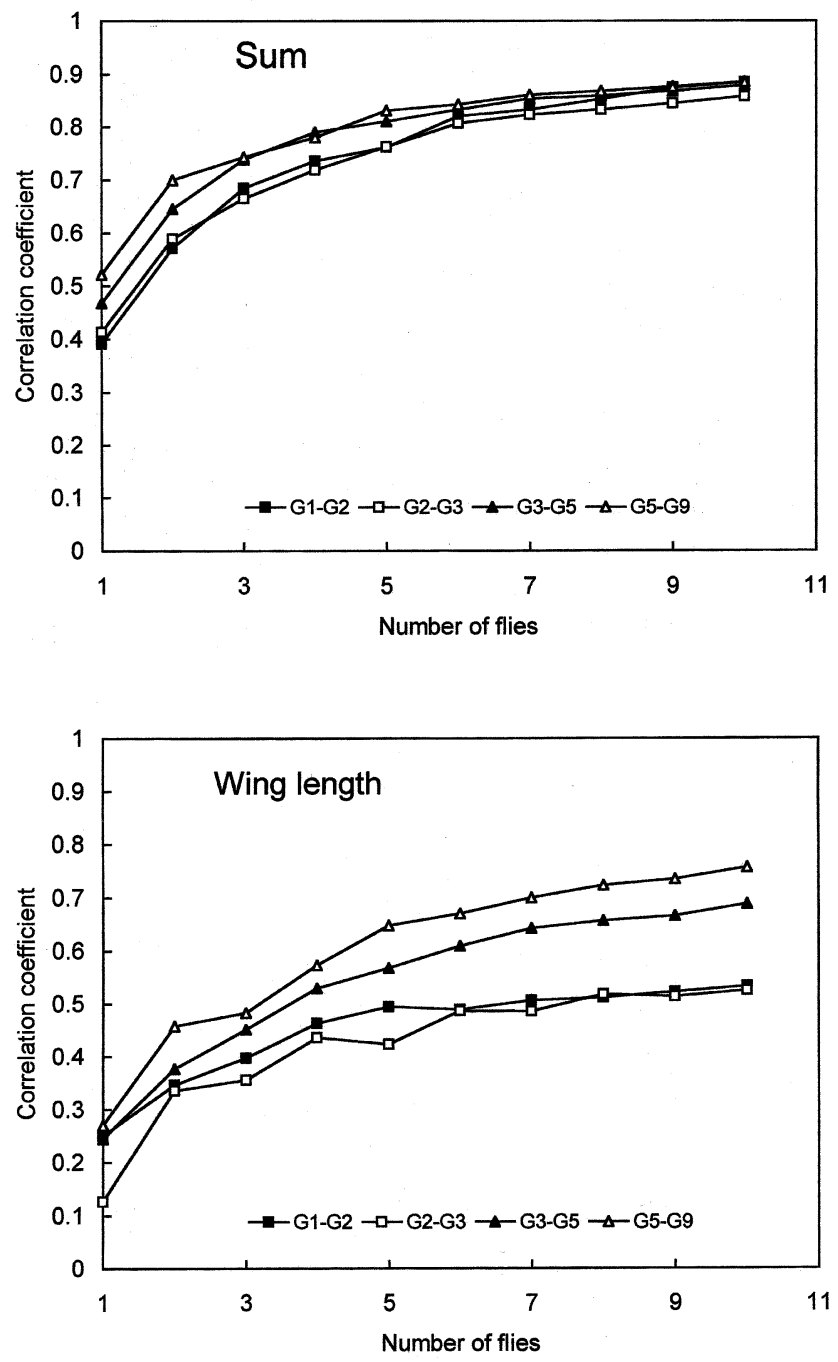

Fig. 3 Variation of the correlation coefficient between family means of laboratory generations of Drosophila melanogaster when increasing the number of flies taken from each family. Four sets of generation pairs were used, as indicated. For each set, 30 repeats were made and values averaged.

Table 6 Average values of wing length CVs from three papers in which several populations of Drosophila melanogaster were investigated both in nature and in the laboratory

\begin{tabular}{lccr}
\hline & Nature & Laboratory & $n$ \\
\hline Coyne \& Beecham (1987) & $5.88 \pm 0.38$ & $3.31 \pm 0.21$ & 10 \\
Imasheva et al. (1994) & $6.60 \pm 0.33$ & $2.95 \pm 0.15$ & 8 \\
Moreteau et al. (1995) & $7.42 \pm 0.31$ & $1.72 \pm 0.06$ & 4 \\
\hline
\end{tabular}

(C) The Genetical Society of Great Britain, Heredity, 80, 326-335. 
study, CV was found to be 7.36 per cent in nature and 2.22 per cent in the laboratory, i.e. a variance about 11 times less in the laboratory. In Coyne \& Beecham's (1987) work, the average ratio was only 3.2; and in Imasheva et al.'s (1994) study it was 5. From Table 6, it appears that average phenotypic variability in natural $D$. melanogaster populations from various parts of the world is quite stable, ranging between 6 per cent and 7 per cent. Laboratory variability, on the other hand, seems to be more heterogeneous among investigators and is more likely to reflect rearing conditions than genetic differences. In several works using a killed yeast, a highly nutritive food, mean CVs ranged from 1.72 (Moreteau et al., 1995) to 2.46 (Capy et al., 1994). In contrast, values obtained by Imasheva et al. (1994) and especially by Coyne \& Beecham (1987) appear significantly higher. This may result, in the latter case, from the use of an instant Drosophila food medium, which must be seeded with live yeast and in which crowding effects are important.

Abdomen pigmentation in wild flies is less documented (Moreteau et al., 1995; present study). Because variability is related to mean value (David et al., 1990), it is better to compare the variances. In the present study, almost identical values were found in nature and in the laboratory for the sum (14.2 vs. 12.8). In Moreteau et al. (1995), variance in nature was twice that found in the laboratory (20.8 against 9.9). These observations suggest that pigmentation is far less influenced by the environmental heterogeneity of natural populations than body size. This is also the case for abdominal bristle number (Coyne \& Beecham, 1987).

A major aim of this paper was to analyse the heritability of quantitative traits, and two methods were used: comparison of wild-living flies with their laboratory progeny and analysis of isofemale lines. For each trait, it was possible to compare successive laboratory generations and to calculate either correlations or regressions.

When individual parents are compared with their progeny, heritability can be estimated by the parentoffspring coefficient of regression. For wing length, a very low and non-significant coefficient $(0.02 \pm 0.03)$ between $\mathrm{P}$ and $\mathrm{G} 1$ was found. Prout (1958) found a negative correlation between wild fathers and laboratory offspring. Coyne \& Beecham (1987) obtained a low positive value for the motherdaughter regression $(0.09 \pm 0.07)$. This reflects the fact that, in nature, the major part of the phenotypic variability is attributable to an environmental, non-heritable component, whereas the additive genetic variance is not significantly increased. In our case, the mother-daughter regression could be extended to the mother-granddaughter, mothergreatgranddaughter, etc. up to the ninth generation. Surprisingly, the regression, which was not significant with G1, became significant with later laboratory generations, providing a highly significant mean value over generations $(0.07 \pm 0.01)$. This value, corresponding to an average heritability of 0.14 , shows that size variations in nature are indeed slightly heritable. Also, Coyne \& Beecham (1987), by investigating both sexes, came to a mean value of 0.22 .

Under laboratory conditions, heritability can be estimated by the coefficient of intraclass correlation (Hoffmann \& Parsons, 1988; Falconer, 1989). For wing length, the average $t$-value was found to be $0.34 \pm 0.03$, close to the average value of 0.40 calculated from many geographical populations (Capy et al., 1994). Under classical theory, $t$ is assumed to correspond to $0.5 h^{2}$ (Falconer, 1989). In practice, $t$ is usually too high, so that $h^{2}<2 t$ (see Capy et al., 1994 for detailed discussion). This may occur from the possible role of dominance and epistatic components in the genetic variance. The founder effect that occurs in each line may initiate some new genetic variation, which will persist in further generations (Capy et al., 1994; Ritchie \& Kyriacou, 1994).

For pigmentation traits, phenotypic variances in nature and in the laboratory were similar, so that no bias was expected in calculating parent-offspring regression. Heritability estimates were higher than for wing length, generally between 0.40 and 0.62 . High values were also found for the intraclass correlations, ranging between 0.22 and 0.47 , in agreement with previous observations (David et al., 1990; Moreteau et al., 1995; Gibert et al., 1996).

Pigmentation is certainly a highly heritable trait, both in nature and in the laboratory. A similar conclusion seems also to be valid for abdominal bristle number (Coyne \& Beecham, 1987). In a recent literature survey, Weigensberg \& Roff (1996) argued that, in most organisms, there were no significant differences between laboratory and field estimates of heritability. Our data agree with that conclusion for pigmentation but not for size. Traits that are very plastic, i.e. very sensitive to environmental fluctuations, are likely to show a lower heritability in nature.

Once isofemale lines are established, it may be interesting to demonstrate that variation observed among them is really heritable and not caused by experimental accidents, such as common environment effects. The demonstration is provided by 
considering the correlation between line mean values of different generations. This correlation, which may be called genetic repeatability, does not provide an estimate of heritability. The regression method between two generations cannot be applied, and it was verified that the major axes of the ellipses of different traits had, on average, a slope close to unity. It was found that the average repeatability $(r=0.55 \pm 0.04)$ for wing length, which has a lower heritability, was significantly less than the repeatability of highly heritable traits, such as abdomen segments 6, 7 and the sum (repeatabilities above 0.80 , see Table 3 ). Also, the optimum family size for demonstrating a genetic repeatability among generations is higher for less heritable traits.

A last and practical conclusion of the present study is that the genetic properties of the isofemale lines remained quite stable over nine generations for all the traits investigated. This stability is best shown by the stability of the overall mean in successive generations. Some evidence exists that the variance between lines and the intraclass correlations increased over time, presumably because of genetic drift, but the effect remained small. Of course, this conclusion applies only to the traits investigated here and to a given method of propagating the lines (killed yeast food and $25^{\circ} \mathrm{C}$ ). Isofemale lines appear to be a convenient way of keeping the quantitative characteristics of a natural population in the laboratory, at least over several months. The possible effects of different environments (e.g. different temperatures) could thus be analysed with excellent precision by keeping subsets of the same lines in different conditions and measuring their characteristics in the same environment periodically.

\section{Acknow ledgement}

We thank Dr A. Gallais for helpful comments on an earlier draft of this paper.

\section{References}

BOCQUET, C., DAVID, J. AND DE SCHEEMAEKER, M. 1973. Variabilité du nombre d'ovarioles des souches sauvages de Drosophila melanogaster conservées en laboratoire sans sélection volontaire. Arch. Zool. Exp. Gen., 114, 475-489.

CAPY, P., PLA, E. AND DAVID, J. R. 1993. Phenotypic and genetic variability of morphometrical traits in natural populations of Drosophila melanogaster and D. simulans. I. Geographic variations. Genet. Sel. Evol., 25, 517-536.

CAPY, P., PLA, E. AND DAVID, J. R. 1994. Phenotypic and genetic variability of morphometrical traits in natural populations of Drosophila melanogaster and D. simulans.
II. Within-population variability. Genet. Sel. Evol., 26, 15-28.

CAVICCHI, S. D., GUERrA, G., GIORGI, G. AND PEZzoli, C. 1985. Temperature-related divergence in experimental populations of Drosophila melanogaster. I. Genetic and developmental basis of wing size and shape variation. Genetics, 109, 665-689.

COYNE, J. A. AND BEECHAM, E. 1987. Heritability of two morphological characters within and among natural populations of Drosophila melanogaster. Genetics, 117, 727-737.

DAVID, J. R. AND CAPY, P. 1988. Genetic variation of Drosophila melanogaster natural populations. Trends Genet., 4, 106-111.

DAVID, J. R., COHET, Y., FOUILlET, P. AND ARENS, M. F. 1980. Phenotypic variability of wild collected Drosophila: an approach toward understanding selective pressures in natural populations. Egyp. J. Genet. Cytol., 9, 51-66.

DAVID, J. R., ALLEMAND, R., VAN HERREWEGE, J. AND COHET, Y. 1983. Ecophysiology: abiotic factors. In: Ashburner, M., Carson, H. L. \& Thompson, J. N., Jr. (eds) The Genetics and Biology of Drosophila, pp. 105-170. Academic Press, London.

DAVID, J. R., CAPY, P., PAYANT, V. AND TSAKAS, S. 1985. Thoracic trident pigmentation in Drosophila melanogaster: differentiation of geographical populations. Genet. Sel. Evol., 17, 211-224.

DAVID, J. R., CAPY, P. AND GAUThIER, J. P. 1990. Abdominal pigmentation and growth temperatures in Drosophila melanogaster: similarities and differences in the norms of reaction of successive segments. J. Evol. Biol., 3, 429-445.

DElPuECH, J. M., CARTON, Y. AND ROUSH, R. T. 1993. Conserving genetic variability of a wild insect population under laboratory conditions. Entomol. exp. appl., 67, 233-239.

DobZhansky, т. 1970. Genetics of the Evolutionary Process. Columbia University Press, New York.

FALCONER, D. S. 1989. Introduction to Quantitative Genetics, 3rd edn. Longman, New York.

GIBERT, P., MORETEAU, B., MORETEAU, J. C. AND DAVID, J. R. 1996. Growth temperature and adult pigmentation in two Drosophila sibling species: a probably adaptive convergence of reaction norms in sympatric populations. Evolution, 50, 2346-2353.

HOFFMAN, A. A. AND PARSONS, P. A. 1991. Evolutionary Genetics and Environmental Stress. Oxford University Press, Oxford.

IMAsheVA, A. G., BUbli, O. A. AND LAZEBNy, O. E. 1994. Variation in wing length in Eurasian populations of Drosophila melanogaster. Heredity, 72, 508-514.

LEWontin, R. C. 1974. The Genetic Basis of Evolutionary Change. Columbia University Press, New York.

MERREL, D. J. 1981. Ecological Genetics. Longman, London.

MORETEAU, B., CAPY, P., ALONSO-MORAGA, A., MUNOZSERRANO, A., STOCKEL, J. AND DAVID, J. R. 1995. Genetic

(C) The Genetical Society of Great Britain, Heredity, 80, 326-335. 
analysis of quantitative traits in natural populations of Drosophila melanogaster: isofemale line vs. isogroups. Genetica, 96, 207-215.

PARSONS, P. A. 1983. The Evolutionary Biology of Colonizing Species. Cambridge University Press, Cambridge.

PARTRIDGE, L., BARRIE, B., FOWLER, K. AND FRENCH, V. 1994. Evolution and development of body size and cell size in Drosophila melanogaster in response to temperature. Evolution, 48, 1269-1276.

PROUT, T. 1958. A possible difference in genetic variance between wild and laboratory populations. Dros. Inf. Serv., 32, 148-149.
RISKA, B., PROUT, T. AND TURELLI, M. 1989. Laboratory estimates of heritabilities and genetic correlations in nature. Genetics, 123, 865-871.

RITCHIE, M. G. AND KYRIACOU, C. P. 1994. Genetic variability of courtship song in a population of Drosophila melanogaster. Anim. Behav., 48, 425-434.

VAN DELDEN, w. 1982. The alcohol dehydrogenase polymorphism in Drosophila melanogaster. Selection at an enzyme locus. Evol. Biol., 15, 187-222.

WEIGENSBERG, I. AND ROFF, D. A. 1996. Natural heritabilities: can they be reliably estimated in the laboratory? Evolution, 50, 2149-2157. 